\title{
Ad-Hoc Reviewers from 2010
}

The Journal of Nonverbal Behavior greatly appreciates the assistance of the Editorial Board as well as the following ad hoc manuscript reviewers:

Reginald Adams

Martha Alibali

Susan Andrzejewski

Charles Areni

Anthony Atikinson

Tanja Banziger

Pascal Belin

Sarai Blincoe

Marc Brackett

Sara Broaders

Jeffrey Cohn

C. Randall Colvin

Martin Corley

Mark Coulson

Timothy DeGroot

Dariusz Dolinski

Sally Farley

Pierre Feyereisen

Agneta Fischer

Steve Gangestad

Maurizio Gentilucci

Pierre Gosselin

Heather Gray

Laura Guerrero

Jamin Halberstadt

James Harnsberger
Lasana T. Harris
Judith Holler
Lawrence Hosman
Susan M. Hughes
Lucy Johnston
Peggy Keller
Spencer D. Kelly
Irene Kimbara
Sotaro Kita
Marina Kouzakova
Eva Gabriele Krumhuber
Elizabeth Kuipers
Jessica Lakin
Anna-Maria Laukkanen
Tera Letzring
Thomas Malloy
Antony Manstead
Valerie Manusov
Abigail Marsh
Florian Menz
L. Steven Miller
Eric Moody
Agnes Moors
Nora A. Murphy

Swann Pichon

Stephen Porter

Gilles Pourtois

Fischer P. Ronald

Erika Rosenberg

Lisa Rubler

Nicholas Rule

Wataru Sato

Tamsin Saxton

Jason Schiffman

Karen L. Schmidt

David Ryan Schurtz

Gwenda Simons

Lisa Slattery Walker

Robert Soussignan

Reiner Sprengelmeyer

Fritz Strack

Thomas Sy

Yolanda van Beek

Max Weisbuch

Sheri Widen

Jamil Zaki

Moshe Zeidner 